
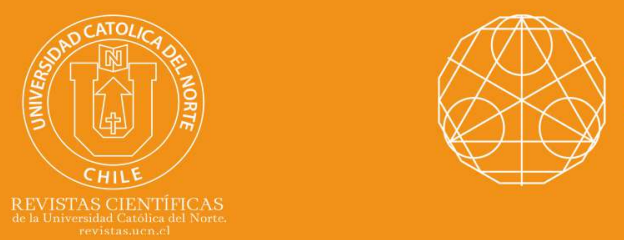

doi"10.22199/issn.0717-6279-2021-01-0005

\title{
Remarks on the mutual singularity of multifractal measures
}

\section{Bilel Selmi ${ }^{1}$ (1) orcid.org/0000-0001-8823-6699}

${ }^{1}$ University of Monastir, Dept. of Mathematics, Analysis, Probability and Fractals Laboratory LR18ES17. Monastir, Tunisia.

घilel.selmi@fsm.rnu.tn

\section{Abstract:}

In the present work, we study the mutual singularity of multifractal Hausdorff and packing measures which provide a positive answer to Olsen's questions in a more general framework. Our main results apply to a family of measures supported by the full 5-adic grid of $[0,1]$, namely the quasiBernoulli measures.

Keywords: Multifractal analysis; Multifractal formalism; Singularity.

MSC (2020): 28A78, 28A80.

\section{Cite this article as (IEEE citation style):}

Bilel Selmi, "Remarks on the mutual singularity of multifractal measures", Proyecciones (Antofagasta, On line), vol. 40, no. 1, pp. 73-84, 2021, doi: 10.22199/issn.0717-6279-2021-010005

Article copyright: (C) 2021 Bilel Selmi. This is an open access article distributed under the terms of the Creative Commons License, which permits unrestricted use and distribution provided the original author and source are credited. 


\section{Introduction and statements of results}

For a long time, the interest of mathematicians in singularly continuous measures and probability distributions was fairly weak, which can be explained, on the one hand, by the absence of adequate analytic apparatus for specification and investigation of these measures, and, on the other hand, by a widespread opinion about the absence of applications of these measures. Due to the fractal explosion and a deep connection between the theory of fractals and singular measures, the situation has radically changed in the last years. It was proved that singular distributions of probabilities are dominant for many classes of random variables. Possible applications in the spectral theory of self-adjoint operators serve as an additional stimulus for a further investigation of singularly continuous measures. The purpose of this paper is to show that the multifractal Hausdorff and packing measures are mutually singular. We first recall the definition of the multifractal Hausdorff measure and the the multifractal packing measure. For an arbitrary Borel probability measure $\mu$ on $\mathbf{R}^{n}$, Olsen introduced two parameter families of measures,

$$
\left\{\mathcal{H}_{\mu}^{q, t} ; q, t \in \mathbf{R}\right\} \quad \text { and } \quad\left\{\mathcal{P}_{\mu}^{q, t} ; q, t \in \mathbf{R}\right\}
$$

based on certain generalizations of the Hausdorff measure and of the packing measure. For $q, t \in \mathbf{R}, E \subseteq \mathbf{R}^{n}$ and $\delta>0$, write

$$
\overline{\mathcal{P}}_{\mu, \delta}^{q, t}(E)=\sup \left\{\sum_{i} \mu\left(B\left(x_{i}, r_{i}\right)\right)^{q}\left(2 r_{i}\right)^{t}\right\}, \quad E \neq \emptyset
$$

where the supremum is taken over all centered $\delta$-packing of $E$. Moreover we can set $\overline{\mathcal{P}}_{\mu, \delta}^{q, t}(\emptyset)=0$. Also, we define

$$
\overline{\mathcal{H}}_{\mu, \delta}^{q, t}(E)=\inf \left\{\sum_{i} \mu\left(B\left(x_{i}, r_{i}\right)\right)^{q}\left(2 r_{i}\right)^{t}\right\}, \quad E \neq \emptyset
$$

where the infinimum is taken over all centered $\delta$-covering of $E$. Moreover we can set $\overline{\mathcal{H}}_{\mu, \delta}^{q, t}(\emptyset)=0$. Especially, we have the conventions $0^{q}=\infty$ for $q \leq 0$ and $0^{q}=0$ for $q>0$.

The packing and Hausdorff pre-measures are defined respectively by

$$
\overline{\mathcal{P}}_{\mu}^{q, t}(E)=\inf _{\delta>0} \overline{\mathcal{P}}_{\mu, \delta}^{q, t}(E) \quad \text { and } \quad \overline{\mathcal{H}}_{\mu}^{q, t}(E)=\sup _{\delta>0} \overline{\mathcal{H}}_{\mu, \delta}^{q, t}(E) .
$$


The function $\overline{\mathcal{P}}_{\mu}^{q, t}$ is not necessarily countably subadditive, also the set function $\overline{\mathcal{H}}_{\mu}^{q, t}$ is not necessarily monotone. For these reasons, Olsen introduced the packing and Hausdorff measures denoted respectively by $\mathcal{P}_{\mu}^{q, t}$ and $\mathcal{H}_{\mu}^{q, t}$ are defined as following

$$
\mathcal{P}_{\mu}^{q, t}(E)=\inf _{E \subseteq \bigcup_{i} E_{i}} \sum_{i} \overline{\mathcal{P}}_{\mu}^{q, t}\left(E_{i}\right) \quad \text { and } \quad \mathcal{H}_{\mu}^{q, t}(E)=\sup _{F \subseteq E} \overline{\mathcal{H}}_{\mu}^{q, t}(F) .
$$

The functions $\mathcal{H}_{\mu}^{q, t}$ and $\mathcal{P}_{\mu}^{q, t}$ are metric outer measures and thus measures on the Borel family of subsets of $\mathbf{R}^{n}$. It is easy to see that $\mathcal{P}_{\mu}^{q, t} \leq \overline{\mathcal{P}}_{\mu}^{q, t}$. Moreover, by using Besicovitch's theorem, there exists an integer $\xi \in \mathbf{N}$, such that $\mathcal{H}_{\mu}^{q, t} \leq \xi \mathcal{P}_{\mu}^{q, t}$ (see [10]). The measure $\mathcal{H}_{\mu}^{q, t}$ is a multifractal generalization of the centered Hausdorff measure, whereas $\mathcal{P}_{\mu}^{q, t}$ is a multifractal generalization of the packing measure. In fact, in the case when $t \geq 0$, $\mathcal{H}_{\mu}^{0, t}=\mathcal{H}^{t}$ and $\mathcal{P}_{\mu}^{0, t}=\mathcal{P}^{t}$, where $\mathcal{H}^{t}$ denotes the t-dimensional centered Hausdorff measure and $\mathcal{P}^{t}$ denotes the t-dimensional packing measure.

The measures $\mathcal{H}_{\mu}^{q, t}$ and $\mathcal{P}_{\mu}^{q, t}$ and the pre-measure $\overline{\mathcal{P}}_{\mu}^{q, t}$ assign in the usual way a multifractal dimension to each subset $E$ of $\mathbf{R}^{n}$. They are respectively denoted by $b_{\mu}^{q}(E), B_{\mu}^{q}(E)$ and $\Lambda_{\mu}^{q}(E)$ and satisfy

$$
\begin{gathered}
b_{\mu}^{q}(E)=\inf \left\{t \in \mathbf{R} ; \quad \mathcal{H}_{\mu}^{q, t}(E)=0\right\}, \quad B_{\mu}^{q}(E)=\inf \left\{t \in \mathbf{R} ; \quad \mathcal{P}_{\mu}^{q, t}(E)=0\right\}, \\
\Lambda_{\mu}^{q}(E)=\inf \left\{t \in \mathbf{R} ; \quad \overline{\mathcal{P}}_{\mu}^{q, t}(E)=0\right\} .
\end{gathered}
$$

The number $b_{\mu}^{q}(E)$ is an obvious multifractal analogue of the Hausdorff dimension $\operatorname{dim}_{H}(E)$ of $E$ whereas $B_{\mu}^{q}(E)$ and $\Lambda_{\mu}^{q}(E)$ are obvious multifractal analogues of the packing dimension $\operatorname{dim}_{P}(E)$ and the pre-packing dimension $\Delta(E)$ of $E$ respectively. In fact, it follows immediately from the definitions that

$$
\operatorname{dim}_{H}(E)=b_{\mu}^{0}(E), \quad \operatorname{dim}_{P}(E)=B_{\mu}^{0}(E) \quad \text { and } \quad \Delta(E)=\Lambda_{\mu}^{0}(E) .
$$

Next, for $q \in \mathbf{R}$, we define the dimension functions $b_{\mu}, B_{\mu}$ and $\Lambda_{\mu}$ by $b_{\mu}(q)=b_{\mu}^{q}(\operatorname{supp} \mu), \quad B_{\mu}(q)=B_{\mu}^{q}(\operatorname{supp} \mu)$ and $\Lambda_{\mu}(q)=\Lambda_{\mu}^{q}(\operatorname{supp} \mu)$. It is well known that the functions $b_{\mu}, B_{\mu}$ and $\Lambda_{\mu}$ are decreasing and $B_{\mu}, \Lambda_{\mu}$ are convex and satisfying $b_{\mu} \leq B_{\mu} \leq \Lambda_{\mu}$.

Multifractal analysis is a natural framework to finely describe geometrically the heterogeneity in the distribution at small scales of the elements 
of compactly supported Borel positive and finite measures on $\mathbf{R}^{n}$. Specifically, this heterogeneity can be described via the lower and upper local dimensions of a measure $\mu$, namely

$$
\underline{\alpha}_{\mu}(x)=\liminf _{r \rightarrow 0} \frac{\log \mu(B(x, r))}{\log r} \quad \text { and } \quad \bar{\alpha}_{\mu}(x)=\limsup _{r \rightarrow 0} \frac{\log \mu(B(x, r))}{\log r} .
$$

When $\underline{\alpha}_{\mu}(x)=\bar{\alpha}_{\mu}(x)$, we refer to the common value as the local dimension of $\mu$ at $x$, and we denote it by $\alpha_{\mu}(x)$. For $\alpha \geq 0$, let us introduce the fractal sets which are also very natural, and the most studied in the literature,

$$
\underline{E}_{\alpha}=\left\{x \in \operatorname{supp} \mu ; \quad \underline{\alpha}_{\mu}(x) \geq \alpha\right\}, \quad \bar{E}^{\alpha}=\left\{x \in \operatorname{supp} \mu ; \quad \bar{\alpha}_{\mu}(x) \leq \alpha\right\}
$$

and

$$
E(\alpha)=\underline{E}_{\alpha} \cap \bar{E}^{\alpha} .
$$

Inspired by the observations made by physicists of turbulence and statistical mechanics, mathematicians derived, and in many situations justified the heuristic claiming that for a measure possessing a self-conformal like property, its Hausdorff spectrum should be obtained as the Legendre transform of a kind of free energy function called $L^{q}$-spectrum. This gave birth to the abundant literature on the so-called multifractal formalisms, which aim at linking the asymptotic statistical properties of a given measure with its fine geometric properties. One of the main importance of the multifractal measures $\mathcal{H}_{\mu}^{q, t}$ and $\mathcal{P}_{\mu}^{q, t}$, and the corresponding dimension functions $b_{\mu}, B_{\mu}$, and $\Lambda_{\mu}$ is due to the fact that the multifractal spectra functions $f_{\mu}$ and $F_{\mu}$ are bounded above by the Legendre transforms of $b_{\mu}$ and $B_{\mu}$, respectively, i.e.,

$$
f_{\mu}:=\operatorname{dim}_{H}(E(\alpha)) \leq b_{\mu}^{*}(\alpha) \quad \text { and } \quad F_{\mu}:=\operatorname{dim}_{P}(E(\alpha)) \leq B_{\mu}^{*}(\alpha)
$$

for all $\alpha \geq 0$.

In general, such a minoration is related to the existence of an auxiliary measure which is supported by the set to be analyzed. Olsen also gives a result in such a way and supposes the existence of a Gibbs' measure at a state $\left(q, B_{\mu}(q)\right)$ for the measure $\mu$, i.e., the existence of a measure $\nu_{q}$ on $\operatorname{supp} \mu$ and constants $\underline{K}, \bar{K}>0$ with $\bar{K}=\underline{K}^{-1}$ and $\delta>0$ such that for every $x \in \operatorname{supp} \mu$ and every $0<r<\delta$,

$$
\underline{K} \mu(B(x, r))^{q}(2 r)^{B_{\mu}(q)} \leq \nu_{q}(B(x, r)) \leq \bar{K} \mu(B(x, r))^{q}(2 r)^{B_{\mu}(q)}
$$


to conclude that

$$
\operatorname{dim}_{H}(E(\alpha))=\operatorname{dim}_{P}(E(\alpha))=b_{\mu}^{*}(\alpha)=B_{\mu}^{*}(\alpha) \text {, where } \quad \alpha=-B_{\mu}^{\prime}(q) .
$$

The authors in $[7,8,10]$ provided some examples of the mutual singularity of multifractal Hausdorff and packing measures for graph directed self-similar measures in $\mathbf{R}^{n}$ with totally disconnected support, cookie-cutter measures [10], for some homogeneous Moran measures [7, 8] and for selfsimilar measures satisfying the significantly weaker open set condition $[5,6]$. The aim of this article is to show that the multifractal Hausdorff and packing measures are mutually singular and provides a positive answer to Olsen's questions [10, Questions 7.1 and 7.2] in a more general setting. Our results apply to a family of measures supported by the full 5 -adic grid of $[0,1]$, namely the quasi-Bernoulli measures.

These more general results are stated as follows:

Theorem 1. Assume that $b_{\mu}=B_{\mu}$ and $B_{\mu}$ is differentiable at $p$ and $q$ with $B_{\mu}^{\prime}(p) \neq B_{\mu}^{\prime}(q)$. Then

$$
\mathcal{H}_{\mu}^{p, b_{\mu}(p)} \perp \mathcal{H}_{\mu}^{q, b_{\mu}(q)} \quad \text { on } \quad \text { supp } \mu .
$$

Theorem 2. Assume there exists a Gibbs measure $\nu_{q}$ for $\mu$ at $\left(q, B_{\mu}(q)\right)$ and $B_{\mu}$ is differentiable at $p$ and $q$. Then, for all $p, q \in \mathbf{R}$ with $B_{\mu}^{\prime}(p) \neq$ $B_{\mu}^{\prime}(q)$ we have

$$
\mathcal{H}_{\mu}^{p, b_{\mu}(p)} \perp \mathcal{H}_{\mu}^{q, b_{\mu}(q)} \quad \text { and } \quad \mathcal{P}_{\mu}^{p, B_{\mu}(p)} \perp \mathcal{P}_{\mu}^{q, B_{\mu}(q)} \quad \text { on } \quad \text { supp } \mu .
$$

Remark 1. The results of Theorems 1 and 2 hold if we replace the multifractal function $B_{\mu}$ by the function $\Lambda_{\mu}$.

\section{Proof of the main results}

\subsection{Proof of Theorem 1}

It follows from [11, Theorem 1.1] that

$$
\lim _{r \downarrow 0} \frac{\log \mu(B(x, r))}{\log r}=-B_{\mu}^{\prime}(q), \mathcal{H}_{\mu}^{q, b_{\mu}(q)}-\text { a.e. }
$$

which implies that $\mathcal{H}_{\mu}^{q, b_{\mu}(q)}\left(E\left(-B_{\mu}^{\prime}(q)\right)\right)=1$. Finally, we conclude that if $p, q \in \mathbf{R}$ with $B_{\mu}^{\prime}(p) \neq B_{\mu}^{\prime}(q)$, then

$$
\mathcal{H}_{\mu}^{p, b_{\mu}(p)} \perp \mathcal{H}_{\mu}^{q, b_{\mu}(q)} \quad \text { on } \quad \operatorname{supp} \mu .
$$




\subsection{Proof of Theorem 2}

We present some tools, as well as lemmas, which will be used in the proof of our main result.

Lemma 1. For any $q \in \mathbf{R}$, we have

$$
\underline{K} \nu_{q} \leq \mathcal{H}_{\mu}^{q, B_{\mu}(q)} \quad \text { on } \operatorname{supp} \mu .
$$

Proof. Fix $\delta>0$ and let $\left(B\left(x_{i}, r_{i}\right)\right)_{i \in \mathbf{N}}$ be a centered $\delta$-covering of $\operatorname{supp} \mu$. Then

$$
\begin{aligned}
\nu_{q}(\operatorname{supp} \mu) & \leq \sum_{i} \nu_{q}\left(B\left(x_{i}, r_{i}\right)\right) \\
& \leq \bar{K} \sum_{i} \mu\left(B\left(x_{i}, r_{i}\right)\right)^{q}\left(2 r_{i}\right)^{B_{\mu}(q)}
\end{aligned}
$$

Consequently

$$
\underline{K} \nu_{q}(\operatorname{supp} \mu) \leq \overline{\mathcal{H}}_{\mu, \delta}^{q, B_{\mu}(q)}(\operatorname{supp} \mu) \leq \overline{\mathcal{H}}_{\mu}^{q, B_{\mu}(q)}(\operatorname{supp} \mu) \leq \mathcal{H}_{\mu}^{q, B_{\mu}(q)}(\operatorname{supp} \mu)
$$

which achieves the proof of Lemma 1.

Lemma 2. For any $q \in \mathbf{R}$, we have

$$
\mathcal{P}_{\mu}^{q, B_{\mu}(q)} \leq \bar{K} \nu_{q} \quad \text { on } \operatorname{supp} \mu .
$$

Proof. Let $F$ be a closed subset of $\operatorname{supp} \mu$. For $\delta>0$ write

$$
B(F, \delta)=\{x \in \operatorname{supp} \mu ; \quad \operatorname{dist}(x, F) \leq \delta\} .
$$

Since $F$ is closed, $B(F, \delta) \searrow F$ for $\delta \searrow 0$. Then for all $\varepsilon>0$, there exists $\delta_{0}$ satisfying

$$
\nu_{q}(B(F, \delta)) \leq \nu_{q}(F)+\varepsilon, \quad \forall 0<\delta<\delta_{0}
$$

Fix $\delta>0$ and let $\left(B\left(x_{i}, r_{i}\right)\right)_{i}$ be a centered $\delta$-packing of $F$. Observing that 


$$
\begin{aligned}
\underline{K} \sum_{i} \mu\left(B\left(x_{i}, r_{i}\right)\right)^{q}\left(2 r_{i}\right)^{B_{\mu}(q)} & \leq \sum_{i} \nu_{q}\left(B\left(x_{i}, r_{i}\right)\right) \\
& \leq \nu_{q}(B(F, \delta)) \leq \nu_{q}(F)+\varepsilon \\
& \leq \nu_{q}(\operatorname{supp} \mu)+\varepsilon .
\end{aligned}
$$

It results that

$$
\overline{\mathcal{P}}_{\mu}^{q, B_{\mu}(q)}(F) \leq \bar{K}\left(\nu_{q}(\operatorname{supp} \mu)+\varepsilon\right) .
$$

Letting $\varepsilon \downarrow 0$, now yields

$$
\mathcal{P}_{\mu}^{q, B_{\mu}(q)}(\operatorname{supp} \mu) \leq \overline{\mathcal{P}}_{\mu}^{q, B_{\mu}(q)}(\operatorname{supp} \mu) \leq \bar{K} \nu_{q}(\operatorname{supp} \mu)
$$

which proves the desired result.

Let us now prove our main theorem. It follows easily from Lemmas 1 and 2 that

$$
\underline{K} \nu_{q} \leq \mathcal{H}_{\mu}^{q, b_{\mu}(q)} \leq \xi \mathcal{P}_{\mu}^{q, B_{\mu}(q)} \leq \xi \bar{K} \nu_{q} \quad \text { on } \quad \operatorname{supp} \mu .
$$

It results that

$$
\frac{1}{\xi \bar{K}} \mathcal{H}_{\mu}^{q, b_{\mu}(q)} \leq \nu_{q} \leq \frac{1}{\underline{K}} \mathcal{H}_{\mu}^{q, b_{\mu}(q)} \quad \text { on } \quad \operatorname{supp} \mu
$$

and

$$
\frac{1}{\bar{K}} \mathcal{P}_{\mu}^{q, B_{\mu}(q)} \leq \nu_{q} \leq \frac{\xi}{\underline{K}} \mathcal{P}_{\mu}^{q, B_{\mu}(q)} \quad \text { on } \quad \operatorname{supp} \mu .
$$

Since $\nu_{q}$ is the Gibbs measure for $\mu$ at $\left(q, B_{\mu}(q)\right)$ and if we assume that $B_{\mu}$ is differentiable, it is shown in $[4,10]$ that

$$
\lim _{r \downarrow 0} \frac{\log \mu(B(x, r))}{\log r}=-B_{\mu}^{\prime}(q), \quad \nu_{q}-\text { a.e., }
$$

this implies that $\nu_{q}\left(E\left(-B_{\mu}^{\prime}(q)\right)\right)=1$. We therefore infer that if $p, q \in \mathbf{R}$ with $B_{\mu}^{\prime}(p) \neq B_{\mu}^{\prime}(q)$, then

$$
\nu_{p} \perp \nu_{q} .
$$

Finally, the result follows from (2.1). 


\section{An example}

Let $\mathcal{F}=\cup_{n \geq 1} \mathcal{F}_{n}$ such that $\mathcal{F}_{n}$ stands for a sequence of the 5-adic intervals. If $x$ belongs to $\left[0,1\left[, I_{n}(x)\right.\right.$ stands for the interval $\mathcal{F}_{n}$ which contains $x$. Now, considering $I=I_{\varepsilon_{1} \varepsilon_{2} \cdots \varepsilon_{n}}$ and $J=I_{\varepsilon_{1}^{\prime} \varepsilon_{2}^{\prime} \cdots \varepsilon_{p}^{\prime}}$, we set

$$
I J=I_{\varepsilon_{1} \varepsilon_{2} \cdots \varepsilon_{n} \varepsilon_{1}^{\prime} \varepsilon_{2}^{\prime} \cdots \varepsilon_{p}^{\prime}} .
$$

A probability measure on $[0,1$ [ is said to be quasi-Bernoulli if there exists $C>0$ such that, for any $I, J \in \mathcal{F}$, one has

$$
\frac{1}{C} \mu(I) \mu(J) \leq \mu(I J) \leq C \mu(I) \mu(J) .
$$

We say that the measure $\mu$ has a strong separation condition if

$$
\begin{gathered}
\mu \in \mathcal{D}:=\{\mu \text { is a quasi-Bernoulli measure with } \\
\left\{\begin{array}{l}
\mu\left(I_{\varepsilon_{1} \varepsilon_{2} \cdots \varepsilon_{n}}\right)=0, \text { if } \exists i \varepsilon_{i} \notin\{1,3\} \\
\mu\left(I_{\varepsilon_{1} \varepsilon_{2} \cdots \varepsilon_{n}}\right) \neq 0, \text { if } \forall i \varepsilon_{i} \in\{1,3\}
\end{array}\right\} .
\end{gathered}
$$

Let $\mu \in \mathcal{D}$, for any $q, t \in \mathbf{R}$, one defines

$\mathcal{K}_{\mu}(q, t)=\lim _{\delta \rightarrow 0} \sup \left\{\sum_{j}^{*} \mu\left(I_{j}\right)^{q}\left|I_{j}\right|^{t} ; \quad I_{j} \in \mathcal{F},\left|I_{j}\right| \leq \delta, I_{i} \cap I_{j}=\emptyset, \forall i \neq j\right\}$,

where the star means that the terms for which $\mu\left(I_{j}\right)=0$ are removed (a convention valid throughout this example), and let

$$
\tau_{\mu}(q)=\sup \left\{t \in \mathbf{R} ; \mathcal{K}_{\mu}(q, t)=+\infty\right\} .
$$

The next lemma compare the function $\tau_{\mu}(q)$ to $\Lambda_{\mu}(q)$.

Lemma 3. One has $\tau_{\mu}(q)=\Lambda_{\mu}(q)$.

Proof. It is clear that if $\mu \in \mathcal{D}$, then

$$
\text { supp } \mu=\bigcap_{n \geq 1} \underset{\substack{\varepsilon_{1} \varepsilon_{2} \cdots \varepsilon_{n} \\ \varepsilon_{i} \in\{1,3\}}}{\bigcup} I_{\varepsilon_{1} \varepsilon_{2} \cdots \varepsilon_{n}} .
$$

For $\delta>0$, let $\left(I_{j}\right)_{j}$ be a $\delta$-packing of $\left[0,1\left[\right.\right.$ and $t>\Lambda_{\mu}(q)$. For any $j$ there exists $n_{j} \in \mathbf{N}^{*}$ such that $I_{j} \in \mathcal{F}_{n_{j}}, x_{j} \in I_{j} \cap K$ and

$$
I_{j} \subseteq B\left(x_{j}, 5^{-n_{j}}\right), \quad \mu\left(I_{j}\right)=\mu\left(B\left(x_{j}, 5^{-n_{j}}\right)\right) .
$$


The strong separation condition implies that $\left(B\left(x_{j}, 5^{-n_{j}}\right)\right)_{j}$ is a $\delta$-packing of $\operatorname{supp} \mu$. Therefore

$$
\begin{aligned}
\sum_{j} \mu\left(I_{j}\right)^{q}\left|I_{j}\right|^{t} & \leq \sum_{j} \mu\left(B\left(x_{j}, 5^{-n_{j}}\right)\right)^{q}\left(2 \times 5^{-n_{j}}\right)^{t} \\
& \leq \overline{\mathcal{P}}_{\mu, \delta}^{q, t}(K)
\end{aligned}
$$

which implies that

$$
\sup \sum_{j} \mu\left(I_{j}\right)^{q}\left|I_{j}\right|^{t} \leq \overline{\mathcal{P}}_{\mu, \delta}^{q, t}(\operatorname{supp} \mu) \quad \text { and } \quad \mathcal{K}_{\mu}(q, t) \leq \overline{\mathcal{P}}_{\mu}^{q, t}(\operatorname{supp} \mu)<+\infty
$$

which proves that $\tau_{\mu}(q) \leq \Lambda_{\mu}(q)$.

The converse inequality is obvious, indeed, let $t$ and $\delta$ be two real numbers such that $t>\tau_{\mu}(q)$ and $\delta>0$. Let $\left(B\left(x_{j}, r_{j}\right)\right)_{j}$ be a $\delta$-packing of $\operatorname{supp} \mu$. Fix $j$, since $x_{j} \in \operatorname{supp} \mu$, there exits $n_{j} \in \mathbf{N}^{*}$ such that

$$
\frac{1}{5^{n_{j}}} \leq r_{j}<\frac{1}{5^{n_{j}-1}}
$$

which implies that

$$
I_{n_{j}}\left(x_{j}\right) \subseteq B\left(x_{j}, r_{j}\right) \quad \text { and } \quad \mu\left(I_{n_{j}}\left(x_{j}\right)\right) \leq \mu\left(B\left(x_{j}, r_{j}\right)\right) .
$$

Also, each $B\left(x_{j}, r_{j}\right)$ is covered by at most three 5 -adic intervals $I_{j, 1}, I_{n_{j}-1}\left(x_{j}\right), I_{j, 2}$. Also, the strong separation condition ensures that

$$
\mu\left(B\left(x_{j}, r_{j}\right)\right) \leq \mu\left(I_{n_{j}-1}\left(x_{j}\right)\right) .
$$

We can see from the construction of measure $\mu$ that, there exists a constant $C>0$ such that

$$
\mu\left(B\left(x_{j}, r_{j}\right)\right) \leq C \mu\left(I_{n_{j}}\left(x_{j}\right)\right) .
$$

It results that, there exists a $\delta$-packing $\left(I_{n_{j}}\right)_{j}$ of $[0,1[$ such that

$$
\mu\left(I_{n_{j}}\right) \leq \mu\left(B\left(x_{j}, r_{j}\right)\right) \leq C \mu\left(I_{n_{j}}\right) .
$$

One has 


$$
\begin{aligned}
\sum_{j} \mu\left(B\left(x_{j}, r_{j}\right)\right)^{q}\left(2 r_{j}\right)^{t} & \leq C_{q, t} \sum_{j} \mu\left(I_{n_{j}}\right)^{q}\left|I_{n_{j}}\right|^{t} \\
& \leq C_{q, t} \sup \sum_{j} \mu\left(I_{n_{j}}\right)^{q}\left|I_{n_{j}}\right|^{t}
\end{aligned}
$$

which implies that

$$
\overline{\mathcal{P}}_{\mu}^{q, t}(\operatorname{supp} \mu) \leq C_{q, t} \mathcal{K}_{\mu}(q, t)<+\infty .
$$

Consequently, we have $\Lambda_{\mu}(q) \leq t$. Finally, we obtain $\Lambda_{\mu}(q) \leq \tau_{\mu}(q)$ and the proof ends.

It is shown in $[3,12]$ that, if $\mu \in \mathcal{D}$ then there exists a measure $\nu_{q}$ such that for all $I \in\left\{I_{\varepsilon_{1} \varepsilon_{2} \cdots \varepsilon_{n}} \in \mathcal{F}_{n} ; \quad \varepsilon_{i} \in\{1,3\}\right\}$,

$$
\frac{1}{K} \mu(I)^{q}|I|^{\tau_{\mu}(q)} \leq \nu_{q}(I) \leq K \mu(I)^{q}|I|^{\tau_{\mu}(q)}, \quad K>0 .
$$

By using Lemma 3 and (3.1), there exist two constants $\underline{K}>0$ and $\bar{K}>0$ such that

$$
\begin{gathered}
\underline{K} \mu(B(x, r))^{q}(2 r)^{\Lambda_{\mu}(q)} \leq \nu_{q}(B(x, r)) \leq \bar{K} \mu(B(x, r))^{q}(2 r)^{\Lambda_{\mu}(q)} \\
\text { for all } x \in \operatorname{supp} \mu \text { and } 0<r<1,
\end{gathered}
$$

Heurteaux in [9] proved that the function $\Lambda_{\mu}$ is differentiable at $q \in \mathbf{R}$. Now, it follows from Remark 1 that, for any $p, q \in \mathbf{R}$ with $\Lambda_{\mu}^{\prime}(p) \neq \Lambda_{\mu}^{\prime}(q)$,

$$
\mathcal{H}_{\mu}^{p, b_{\mu}(p)} \perp \mathcal{H}_{\mu}^{q, b_{\mu}(q)} \quad \text { and } \quad \mathcal{P}_{\mu}^{p, B_{\mu}(p)} \perp \mathcal{P}_{\mu}^{q, B_{\mu}(q)} \quad \text { on } \quad \operatorname{supp} \mu .
$$

Remark 2. 1. Our main results have been proved rigorously for graph directed self-similar measures in $\mathbf{R}^{n}$ with totally disconnected support, cookie-cutter measures [10], for some homogeneous Moran measures $[7,8]$ and for self-similar measures satisfying the significantly weaker open set condition [5, 6]. Also, these results hold for Mandelbrot measures (see [3] for the definition).

2. The results of Theorems 1 and 2 hold if we replace the multifractal Hausdorff and packing measures by the multifractal Hewitt-Stromberg measures, see [1, 2] for the precise definitions. 


\section{References}

[1] N. Attia and B. Selmi, "Regularities of multifractal Hewitt-Stromberg measures", Communications Korean Mathematics Society, vol. 34, no. 1, pp. 213-230, Jan. 2019, doi: 10.4134/ CKMS.c1 80030

[2] N. Attia and B. Selmi, "A multifractal formalism for Hewitt-Stromberg measures", The journal of geometric analysis, Oct. 2019, doi: $10.1007 / \mathrm{s} 12220-019-00302-3$

[3] J. Barral, F. B. Nasr, and J. Peyriere, "Comparing multifractal formalisms: the neighboring boxes condition", Asian journal of mathematics, vol. 7, no. 2, pp. 149-166, Jan. 2003, doi: 10.4310/ AJM.2003.v7.n2.a1

[4] F. Ben Nasr, I. Bhouri, and Y. Heurteaux, "The validity of the multifractal formalism: results and examples", Advances in mathematics, vol. 165, no.2, pp. 264-284, Feb. 2002, doi: 10.1006/ aima.2001.2025

[5] M. Das, "Pointwise Local Dimensions", Ph. D. Thesis, The Ohio State University, 1996.

[6] M. Das, "Hausdorff measures, dimensions and mutual singularity", Transactions of the American Mathematical Society, vol. 357, no. 11, pp. 4249-4268, Jun. 2005, doi: 10.1090/ S0002-9947-05-04031-6

[7] Z. Douzi and B. Selmi, "On the mutual singularity of multifractal measures", Electronic research archive, vol. 28, no. 1, pp. 423-432, 2020, doi: $10.3934 /$ era. 2020024

[8] Z. Douzi, A. Samti, and B. Selmi, "Another example of the mutual singularity of multifractal measures", Proyecciones (Antofagasta, On line), vol. 40, no. 1, pp. 17-33, Feb. 2021, doi: $10.22199 /$ issn.0717-6279-2021-01-0002

[9] Y. Heurteaux, "Estimations de la dimension inférieure et de la dimension supérieure des mesures", Annales de l'Institut Henri Poincare (B) Probability and Statistics, vol. 34, no. 3, pp. 309-338, 1998, doi: 10.1016/S0246-0203(98)80014-9

[10] L. Olsen, "A multifractal formalism", Advances in mathematics, vol. 116, no. 1, pp. 82-196, Nov. 1995., doi: 10.1006/ aima.1995.1066

[11] L. Olsen, "Dimension inequalities of multifractal Hausdorff measures and multifractal packing measures", Mathematica scandinavica, vol. 86, no. 1, pp. 109-129, Mar. 2000, doi: 10.7146/ math.scand.a-14284 
[12] J. Peyrière, "Multifractal measures", in Probabilistic and stochastic methods in analysis, with applications, J. S. Byrnes, J. L. Byrnes, K. A. Hargreaves, and K. Berry, Eds. Dordrecht: Springer, 1992, pp. 175-186 